\title{
Undulator-Like Radiation and Cooperative Phenomenon in Semiconductor Microstructure with Grating
}

\author{
I. TRALlE* AND P. ZiĘBA \\ Institute of Physics, Mathematics and Natural Sciences Department, University of Rzeszów \\ al. T. Rejtana 16A, 35-310 Rzeszów, Poland
}

\begin{abstract}
In the article the cooperative $N^{2}$-effect is considered, that is the radiation whose power is $\sim N^{2}$, where $N$ is the number of emitters which in this case is equal to the number of electrons in a bunch. The suggested effect is the result of combining two other effects, Gunn-effect in GaAs and undulator-like radiation which can be produced by means of microstructure with grating (microundulator). It is very probable that the effect can be used for the developing of a new semiconductor-based room temperature source of the $\mathrm{THz}$ radiation.
\end{abstract}

PACS: 41.60.-m, 42.50.Gy, 73.61.Ey, 73.40.kp, 73.50.Fq

\section{Introduction}

Nowadays there is a growing interest in developing the new sources of $\mathrm{THz}$ radiation because of variety of its possible applications ranging from security service [1] to biochemistry and medicine [2]. There are many propositions concerning possible schemes of $\mathrm{THz}$ generation/ detection, broad-band as well as narrow-band, based on optical rectification, photoconductive effect, parametric conversion etc. (for the review see $[3,4]$ ). Each of the proposed devices has its own advantages as well as the limitations of its use. For example, some of them require cryogenic cooling, and until the sources require this cooling, it limits greatly their possible application. Recently the development of the room temperature terahertz quantum cascade laser was reported [5]. It seems however, that the existing modern fabrication techniques, such as molecular beam epitaxy combined with ultrahigh-resolution electron beam lithography also enable to develop another type of room temparature sources of $\mathrm{THz}$ radiation, based on the "microundulator" which would be able to generate in this spectral region.

Suppose we have a microstructure very similar to that reported in [6], which was grown on a semi-insulating GaAs substrate using molecular beam epitaxy, but differs from it in the respect that instead of two electrodes on the sample surface there are multiple electrodes on both sides of it, top and bottom (see Fig. 1), which create gratings. If the electric bias is applied to the electrodes, then a weak periodic potential modulation arises within the GaAs-sample and this periodic electric field becomes very similar to the periodic electric field used in some types of undulators (in most of the cases in undulators the periodic arrangement of magnets is used, however the periodic electric field is also employed, especially

* corresponding author; e-mail: tralle@univ.rzeszow.pl

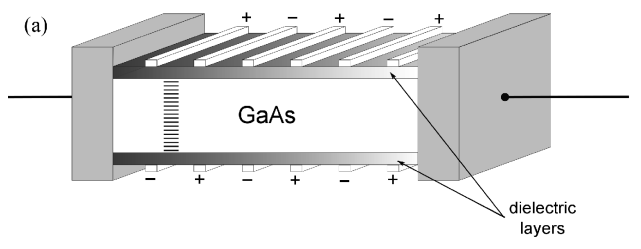

(b)

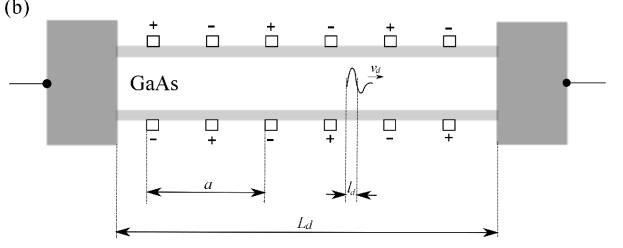

Fig. 1. A sketch (a) and the parameters (b) of generic microstructure (microundulator). The symbols are: $L_{d}$ - the sample length, $l_{d}$ - the thickness of the bunch, $v_{d}$ - bunch velocity, $a-$ the period of grating (as for numerical values of parameters, see the text).

in free electron lasers (FEL)). The radiation field produced by the charged particles moving in such structure should consist of narrow spectral lines whose frequencies are $\omega=2 \pi m T^{-1} /\left(1-\frac{L}{c T} \cos \vartheta\right)$. Here $T=L / v$, where $v$ is the charges' velocity, $L$ - the spacing of grating (or the period of potential modulation), $c$ - the speed of light, $m$ - an integer and $\vartheta$ - the observation (excursion) angle. This is exactly the radiation spectrum emitted by the particles in an undulator.

The spectrum described by the formulae mentioned above does not depend on whether the periodic oscillations of velocity are caused by periodic magnetic or periodic electric field, since the situation in both cases looks very similar and, in a sense, the two cases are dual. Indeed, let us suppose that transversal oscillations of an electron's velocity is caused by the periodic arrangement of magnets. Then, in a reference frame where electron is at rest on average, the undulator field has not only mag- 
netic, but also the transverse electric component. That is, it looks like the electromagnetic "pumping wave", while in case of the periodic arrangement of electric field, the undulator field has the transversal magnetic component, looking thus again like electromagnetic "pumping wave".

It is clear that in the proposed structure not an ideal narrow spectral line would be observed, but the spectrum of finite width. Since the electrons undergo periodic oscillations in the structure in question, the generated field consists of a wave train with the relative width of about $\Delta \omega / \omega \sim 1 / m$ where $m$ is the number of periods of the structure. Another source of the spectrum broadening is of course the thermal variations of electrons' velocities. An interesting question is: whether one should take into account the higher harmonics of radiation or it is sufficient to consider only the lowest one? It turns out that if $v \ll c$ (remember, the electron velocity is not greater than $\left.10^{7} \mathrm{~cm} \mathrm{~s}^{-1}\right)$, the highest harmonics can be neglected. Another argument in favour of considering only the lowest harmonics is the following. It is well known that if a function of a single variable $f(t)$ is differentiable everywhere within some closed interval and its first derivative is a function of bounded variance, then the coefficients of the Fourier series for $f(t)$ decrease as their index $n$ increases, at least as $n^{-2}$ (see [7]). If instead, the smoothness of the function $f(t)$ is of a higher order, then the Fourier coefficients can decrease even exponentially.

It seems also that the structure can be used to produce and observe another interesting effect, namely the cooperative $N^{2}$-effect, the coherent radiation with the intensity proportional to $N^{2}$ at the frequencies of about $3 \times 10^{11} \mathrm{~Hz}$, where $N$ is the number of electrons in an electron bunch. The aim of this paper is just to show and discuss how it can be done.

\section{Cooperative $N^{2}$-effect in an ensemble of classical oscillators}

The effect results in the radiation intensity proportional to $N^{2}$, where $N$ is the number of emitters, and is due to specific phase correlations arising in the ensemble of radiating atoms. Perhaps the first person who mentioned such a possibility was Schiff [8]. He noticed that if $N$ particles moving around the circle have the same speed and instantaneous angular position $\varphi_{s}$, then formula for radiation intensity contains the factor $\left|\sum_{s=1}^{N} \exp \left(\mathrm{i} m \varphi_{s}\right)\right|^{2}$, where $m$ is an integer. If the particles are distributed at random around the orbit, this factor is equal to $N$ and radiation intensity per particle is independent of the other particles. If however particles are spaced equally around the circle, the expression above gives zero unless $m / N$ is an integer, in which case it is equal to $N^{2}$.

Particles' movement in a periodic potential formally is similar to the circular motion and hence, one can state that if the ensemble of $N$ identical oscillators (the source of radiation) emits waves, the amplitudes of generated fields being seen at some distance from the source, can add together in a different way, depending on their relative phases. In general for more than one emitter, each emitter radiates with its own phase, and these phases are completely random with respect to one another. The total emitted field is $\boldsymbol{E}_{\mathrm{t}}=\boldsymbol{E}_{\mathrm{t}}^{+}+\boldsymbol{E}_{\mathrm{t}}^{-}$, where

$$
\boldsymbol{E}_{\mathrm{t}}^{+}=\sum_{r=1}^{N} \boldsymbol{E}_{r}^{+} \exp \left(\mathrm{i} \varphi_{r}\right)
$$

and the sum runs over all emitters in the system. The total radiated intensity

$$
\begin{aligned}
I_{\mathrm{t}} & =\left|\sum_{r=1}^{N} \boldsymbol{E}_{r}^{+} \exp \left(\mathrm{i} \varphi_{r}\right)\right|^{2}=\sum_{r=1}^{N}\left|\boldsymbol{E}_{r}^{+}\right|^{2} \\
& +\sum_{r \neq s} \boldsymbol{E}_{r}^{-} \boldsymbol{E}_{s}^{+} \exp \left(-\mathrm{i}\left(\varphi_{r}-\varphi_{s}\right)\right) .
\end{aligned}
$$

Assuming that the amplitudes of the fields emitted by each oscillator are the same $\left|\boldsymbol{E}_{r}\right|^{2}=I$, we get

$$
I_{\mathrm{t}}=N I+I \sum_{r \neq s} \exp \left(-\mathrm{i}\left(\varphi_{r}-\varphi_{s}\right)\right) \text {. }
$$

For random phases, the second term in the expression above averages to zero, that is the total intensity is just the sum of individual intensities: $I_{\mathrm{t}}=N I$. If however, we could somehow force oscillators to emit with roughly the same phase, $\varphi_{r} \approx \varphi_{s}$, for all $r$ and $s$, then $I_{\mathrm{t}}=N I+N(N-1) I \sim N^{2} I$, since usually $N \gg 1$. From now on we shall term the proportionality of radiated intensity to $N^{2}$ as $N^{2}$-effect for short.

The possibility to get the generated power $\sim N^{2}$ was discussed many times in scientific literature and in different contexts. For example, the similar problem appears in discussing the operation modes of FEL (see for instance, [9]). It was suggested many years ago that the specific phase coherence which leads to $\sim N^{2}$-effect can be achieved, if the linear sizes $l$ of the electron bunch in FEL is much smaller than the emitted wavelength $\lambda$. Intuitively it is appealing, quite clear and seems to be almost obvious that if $l \ll \lambda$, the electron bunch generates waves as a single point source, that is, all electrons in a bunch generate waves of the same phase, and since the total charge of a bunch is equal to $N e$, the generated power is $\sim N^{2}$. But if FEL is to generate visible light, to create a bunch whose linear size is of a wavelength then it is, mildly speaking, not a trivial task. It seems however, that to produce a bunch whose linear size is much smaller than the wavelength corresponding to $\mathrm{THz}$ region is quite possible and we are discussing this possibility in the next section.

\section{Gunn-effect and undulator-like radiation}

Suppose we have GaAs sample normally used for the fabrication of the Gunn-effect diodes and assume that this Gunn-effect diode structure is equipped with the gratings similar to that presented in Fig. 1. Then, due to the gratings on the top and bottom of the structure there 
will be a weak periodic potential modulation within the semicondutor.

Suppose now that all other conditions necessary for the Gunn-effect to appear are fulfilled. Then, the strong electric field domain moving within the sample is accompanied by the electron "bunch", whose electron concentration is greater than some threshold concentration and which can be estimated at about $10^{16} \mathrm{~cm}^{-3}$. The thickness of the "bunch" $l_{d}$ ranges from $1 / 10$ to $1 / 30$ of the sample length $L_{d}$. If we suppose the length of the Gunn-efect diode $L_{d}$ to be equal to $10^{-3} \mathrm{~cm}$, then the thickness of a bunch can be estimated to be $\approx 3 \times 10^{-5} \mathrm{~cm}$. If we suppose the period of grating $a$ on a top and bottom of the structure to be about $2 \times 10^{-4} \mathrm{~cm}$, the frequency of the undulator-like radiation produced by the structure, will be about $\omega_{0} \approx \frac{2 \pi v_{d}}{a} /\left(1-v_{d} / c\right) \approx$ $3.14 \times 10^{11} \mathrm{~Hz}$. (Here we take into account that the speed of the strong field domain $v_{d}$ is about $10^{7} \mathrm{~cm} \mathrm{~s}^{-1}$ and hence, $v_{d} / c \approx 10^{-3}$ and radiation frequency practically does not depend on the excursion angle $\vartheta$.) The corresponding wavelength is about $\lambda \approx 0.1816 \mathrm{~cm}$ and obviously, $l_{d} \ll \lambda$.

If the velocity of electrons in a bunch is ultra-relativistic as in case of FEL or in general, in the undulators, the last condition is sufficient for the radiation power to be $\sim N^{2}$. But since in our case the velocity of electrons is much smaller than $c$, to take into account that the size of the bunch has to be much smaller than the period of grating $l_{d} \ll a$ is even more important than to take into account the condition $l_{d} \ll \lambda$, as it will be shown in some of the next sections. The last one guarantees that electrons in the bunch will generate roughly with the same phase.

Now we have all reasons to believe that the electron "bunch", that is the domain of high electron concentration accompanying the strong field domain in the Gunn-effect diode, will generate as a point source. Then the structure in question will generate the pulses of radiation, whose intensity $\sim N^{2}$

\section{Undulator-like radiation and cooperative $N^{2}$-effect}

Until now we have discussed the $\sim N^{2}$-effect from the perspective of making undulator-like radiation coherent due to the fact that the linear size of electron bunch in the Gunn-effect diode is much smaller than the wavelength $\lambda$ of the generated radiation. This point of view makes the situation very similar to the one which takes place in FEL, since the undulator periodic structure is the basic element of FEL.

Let us analyze now the effect which is discussed, from the perspective of the coherence arising in the ensemble of classical oscillators. Of course, the question is to what extent the classical model is an adequate one in this case? It is possible to demonstrate, however, that classical theory works quite well in this situation and as a matter of fact, is sufficient enough to describe it. The arguments in favour of this statement are the following.
First, neither the Gunn-effect nor undulator radiation do not need quantum theory to be properly understood.

Second, compare $\epsilon_{\mathrm{ph}}$, the energy of a single photon emitted by each of the electrons of the domain, with its mean kinetic energy $\epsilon_{\mathrm{k}}$. Assuming the speed of the moving strong field domain to be equal, as previously, $\approx 10^{7} \mathrm{~cm} \mathrm{~s}^{-1}$, electron effective mass corresponding to the "heavy valley" of GaAs conduction band as to be $1.2 m_{\mathrm{e}}\left(m_{\mathrm{e}}\right.$ is free electron mass $)$ and the radiation frequency to be $\omega \approx 3.14 \times 10^{11} \mathrm{~Hz}$, one can easily obtain the value of ratio $\epsilon_{\mathrm{ph}} / \epsilon_{\mathrm{k}} \approx 0.006 \ll 1$. The last argument whatsoever indirect, appeals to an idea taken from the laser theory. It is known that there are great many approaches in a laser theory, in some of them an active medium is treated as the quantum-mechanical ensemble while the radiation as a classical electromagnetic field (see, for instance, [10]), in some others, both atoms and field, are treated quantum-mechanically [11, 12]. However, it turns out and it was shown by Borenstein and Lamb [13] that laser action can be described perfectly well in classical terms only. Hence, to our mind the process of electromagnetic field generation in the structure in question can also be considered in the frame of classical model.

\section{The role of phasing}

In this section we would like to present some evidence in favour of our statement (see Sect. 3) concerning the importance of the condition $l_{d} \ll a$ for $N^{2}$-effect to appear. Let us consider the system of $N$ classical nonlinear oscillators when each one is characterised by mass $m$, charge $e$, some characteristic length $a$ which we associate with a period of grating and the undumped angular frequency $\omega$ in the presence of an external forcing field with the angular frequency $\nu$. Our model is similar to the one proposed in [13], but differs from it by taking into account the radiation damping, then the evolution of the ensemble of nonlinear oscillators is described by the system of equations

$$
\ddot{x}_{i}+\omega^{2} x_{i}=f\left(x_{i}, \dot{x}_{1}, \ldots, \dot{x}_{N}, t\right),
$$

for $i=1, \ldots, N$, where

$$
\begin{aligned}
f & \left(x_{i}, \dot{x}_{1}, \ldots, \dot{x}_{N}, t\right) \\
& =\frac{1}{6} \frac{\omega^{2}}{a^{2}} x_{i}^{3}-\frac{2 e^{2} \omega^{2}}{3 m c^{3}} \sum_{j=1}^{N} \dot{x}_{j}+\frac{e E}{m} \cos (\nu t) .
\end{aligned}
$$

The first term on the right-hand side is responsible for nonlinearity, since we do not assume the displacements to be small, they can be arbitrary. The second term represents radiation damping of electrons and the third one is nothing else but an external driving field associated with emitted undulator-like radiation whose amplitude is $E$.

As we will see below, each term in the right-hand side is small compared to the terms in the left-hand side and it is possible, by using the slowly varying amplitude and phase method [14], to transform the initial system of $N$ 
nonlinear differential equations of the second order into the system of $2 N$ nonlinear first order differential equations in the amplitude and phase variables. We seek a solution $x_{i}(t)$ to the system of Eqs. (1) in the form

$$
x_{i}(t)=a A_{i}(t) \cos \left(\nu t+\theta_{i}(t)\right),
$$

where by hypothesis $A_{i}(t)$ and $\theta_{i}(t)$ are the functions whose rate of variation is small compared with the angular frequency $\nu$. Differentiating (2) we get

$$
\dot{x}_{i}=a\left[\dot{A}_{i} \cos \left(\nu t+\theta_{i}\right)-A_{i}\left(\nu+\dot{\theta}_{i}\right) \sin \left(\nu t+\theta_{i}\right)\right] .
$$

If the functions $A_{i}, \theta_{i}$ were constants, then the derivatives $\dot{x}_{i}$ would be

$$
\dot{x}_{i}=a \nu A_{i} \sin \left(\nu t+\theta_{i}\right) .
$$

Comparing the last two equations gives

$$
\dot{A}_{i} \cos \left(\nu t+\theta_{i}\right)-A_{i} \dot{\theta}_{i} \sin \left(\nu t+\theta_{i}\right)=0 .
$$

Differentiating (2) twice we compute $\ddot{x}_{i}$ :

$$
\begin{aligned}
\ddot{x}_{i} & =-a \nu\left[\dot{A}_{i} \sin \left(\nu t+\theta_{i}\right)\right. \\
& \left.+A_{i}\left(\nu+\theta_{i}\right) \cos \left(\nu t+\theta_{i}\right)\right] .
\end{aligned}
$$

Inserting (2), (3) and (5) into (1) we get

$$
\begin{aligned}
& \nu \dot{A}_{i} \sin \left(\nu t+\theta_{i}\right)+A_{i}\left[\left(\nu^{2}-\omega^{2}\right)+\nu \dot{\theta}_{i}\right] \cos \left(\nu t+\theta_{i}\right) \\
& \quad=f\left(x_{i}, \dot{x}_{1}, \ldots, \dot{x}_{N}, t\right) / \nu .
\end{aligned}
$$

From (4) and (6) we compute the derivatives $\dot{A}_{i}, \dot{\theta}_{i}$ :

$$
\begin{aligned}
\dot{A}_{i} & =-\frac{1}{\nu^{2}}\left[A_{i}\left(\nu^{2}-\omega^{2}\right) \cos \left(\nu t+\theta_{i}\right)\right. \\
& \left.-f\left(x_{i}, \dot{x}_{1}, \ldots, \dot{x}_{N}, t\right)\right] \sin \left(\nu t+\theta_{i}\right) \\
\dot{\theta}_{i} & =-\frac{\nu^{2}-\omega^{2}}{\nu} \cos ^{2}\left(\nu t+\theta_{i}\right)+\frac{f\left(x_{i}, \dot{x}_{1}, \ldots, \dot{x}_{N}, t\right)}{\nu^{2} A_{i}} .
\end{aligned}
$$

Because $A_{i}$ and $\theta_{i}$ by assumption are slowly varying functions, we substitute the right-hand sides of the last two equations by their values averaged over the period $2 \pi / \nu$. Finally, the system (1) takes the form

$$
\begin{aligned}
& \frac{\mathrm{d} A_{i}}{\mathrm{~d} \tau}=H\left[\sum_{j=1}^{N} A_{j}(\tau) \cos \left(\theta_{i}(\tau)-\theta_{j}(\tau)\right)\right]-G \sin \theta_{i}(\tau), \\
& \frac{\mathrm{d} \theta_{i}}{\mathrm{~d} \tau}=-\frac{1}{2} \frac{\nu^{2}-\omega^{2}}{\nu^{2}}-\frac{1}{16} \frac{\omega^{2}}{\nu^{2}} A_{i}^{2}(\tau)-\frac{G}{A_{i}(\tau)} \cos \theta_{i}(\tau),
\end{aligned}
$$

where

$$
\tau=\nu t, \quad H=\frac{e^{2} \omega^{2}}{3 \nu m c^{3}}, \quad G=\frac{e E}{2 a m \nu^{2}} .
$$

Equations (9), (10) can be solved numerically. In our calculations we set $c=3 \times 10^{10} \mathrm{~cm} / \mathrm{s}, e=4.0832 \times$ $10^{-10} \mathrm{cgs}$ units, $m=1.2 \times 0.91 \times 10^{-28} \mathrm{~g}$ (effective mass of an electron in the "heavy valley" of GaAs-conduction band), $\omega=\nu=3.14 \times 10^{11} \mathrm{~Hz}, E \approx 4 \mathrm{~V} / \mathrm{cm}=0.0135 \mathrm{cgs}$ units (assuming one hundredth of electron energy in a Gunn diode will be converted into generated radiation), $a=2 \times 10^{-4} \mathrm{~cm}, N=10^{4}, G=0.0015$, and $H=$ $8.18 \times 10^{-12}$.

Figures 2 and 3 present the solutions of Eqs. (9), (10) in a series of phase diagrams where $A_{i}(\tau)$ is plotted as a function of $\theta_{i}(\tau)$ for the consecutive moments of dimensionless "time" $\tau=0,25,50,75,100$ for two cases: the first one, when initial phases of individual oscillators are uniformly distributed ranging from 0 to $2 \pi$, and the second one, when initial phases are clustered around $\pi / 4$ with the dispersion 0.15 .

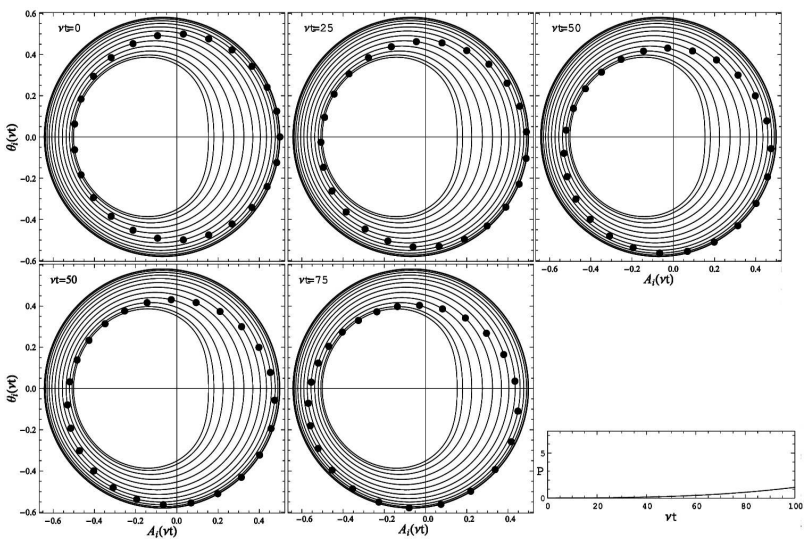

Fig. 2. Phase portrait in case of initial phases of individual oscillators uniformly distributed in the interval from 0 to $2 \pi$. Individual solid lines represent trajectories for different values of initial phase. Black dots indicate oscillators.

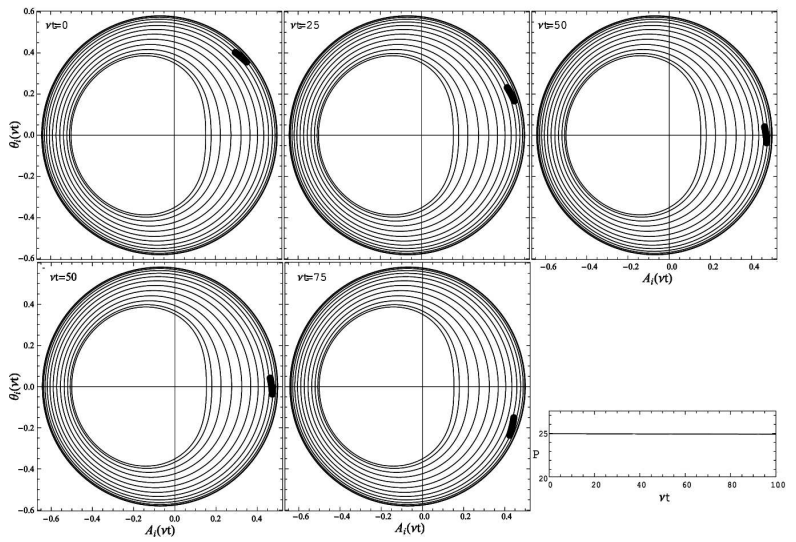

Fig. 3. Phase portrait in case of initial phases clustered around $\pi / 4$ with the dispersion 0.15 . Individual solid lines represent trajectories for different values of initial phase. Cluster of black dots indicates oscillators.

Obviously, the first case corresponds to the situation when $l_{d} \geq a$, while the second one, when $l_{d} / a=0.15$. In both cases initial amplitudes $A_{i}(0)$ for all oscillators are taken to be equal to 0.5. Phase diagrams are accompanied by the plot of a function $P(\tau)$, which is a measure 
of phasing of the electrons in a bunch, defined as:

$$
P(\tau)=\frac{1}{N}\left|\sum_{j=1}^{N} \exp \theta_{j}(\tau)\right|^{2} .
$$

It ranges from 0 when all oscillators have completely random phases, to $N$ when all of them oscillate with roughly the same phase. We conclude that in the first case the driving field is too small to cause phasing of oscillators.

Analysing the second case, we set the upper bound of the sequence of moments of "time" as to be 100; that corresponds to the time needed for an electron bunch to flight through a Gunn diode.

In Figs. 2 and 3 we have shown the results of calculations obtained by using the number of oscillators $N=25$, because at the greater number of oscillators the trajectories become too dense for the phase portraits to be readable and clear. However, it should be noted that with a greater number of oscillators taken into consideration, the picture remains the same in its general features. This is because even if one assumes $N=10^{7}$, the first term on the right side of Eq. (9) (the only term dependent on $N$ ) will be by far smaller as compared to other terms even in case of the most favorable conditions when all phases $\theta_{i}$ coincide.

\section{Conclusion}

In this paper we have considered cooperative $N^{2}$-effect which to our mind, can occur in the GaAs-structure with grating due to combining two other effects, namely the Gunn-effect and the undulator-like radiation. The mechanism which leads to the proportionality of radiation power to $N^{2}$ is the initial phasing of electrons in a bunch due to the Gunn effect and the fact that linear size of a bunch $l_{d}$ is much smaller than the period of grating $a\left(l_{d} \ll a\right)$. As it is known, the length of the Gunn-efect diode $L_{d}$ ranges from $10^{-2}$ to $10^{-3} \mathrm{~cm}$; and the thickness of the bunch $l_{d} \sim L_{d} / 30$. Assuming $L_{d} \approx$ $10^{-3} \mathrm{~cm}$ the thickness of the bunch will be estimated as $l_{d} \sim 3 \times 10^{-5} \mathrm{~cm}$. In order to fulfil the condition $l_{d} \ll a$ we need to have $a \sim 10^{-4} \mathrm{~cm}$. Then, the frequency of the undulator-like radiation produced by the structure will be about $\omega_{0} \approx \frac{2 \pi v_{d}}{a} /\left(1-v_{d} / c\right) \approx 3.14 \times 10^{11} \mathrm{~Hz}$.
It seems very probable that the predicted effect can be used for the developing of generators which could produce radiation at the frequencies up to $\mathrm{THz}$ at room temperature. A general concept presented here describes the main physical phenomena underlying the operation principle of this kind of devices.

\section{Acknowledgments}

The authors are greatly acknowledged to Prof. Jerzy Łusakowski for very fruitful discussions and to the anonymous referee for valuable comments which contributed to the amendment of the clarity of exposition.

\section{References}

[1] A.G. Davies, A.D. Burnett, E.H. Linfield, W. Fan, J.E. Cunningham, Mater. Today 11, 18 (2008).

[2] B.S. Alexandrov, V. Gelev, A.R. Bishop, A. Usheva, K.O. Rasmussen, Phys. Lett. A 374, 1214 (2010).

[3] D. Dragoman, M. Dragoman, Prog. Quant. Electr. 28, 1 (2004).

[4] Yun-Shik Lee, Principles of Terahertz Science and Technology, Springer Science, New York 2009.

[5] M. Belkin, F. Capasso, Xie Feng, A. Belyanin, M. Fischer, A. Wittman, J. Faist, Appl. Phys. Lett. 92, 201101 (2008).

[6] S.Y. Chou, D.R. Allee, R.F.W. Pease, J.S. Harris, Appl. Phys. Lett. 55, 176 (1989).

[7] H. Jeffreys, B. Swirles, Methods of Mathematical Physics, 3rd ed., Cambridge University Press, Cambridge 1966.

[8] L.I. Schiff, Rev. Sci. Instrum. 17, 6 (1946).

[9] P. Meystre, M. Sargent, III, Elements of Quantum Optics, Springer-Verlag, Berlin 2007.

[10] W.E. Lamb, Phys. Rev. 134, A1429 (1964).

[11] M. Scully, W.E. Lamb, Phys. Rev. Lett. 16, 853 (1966).

[12] M. Scully, W.E. Lamb, Phys. Rev. 159, 208 (1967).

[13] M. Borenstein, W.E. Lamb Jr., Phys. Rev. A 5, 1298 (1972).

[14] N.W. McLachlan, Ordinary Non-linear Differential Equations in Engineering and Physical Sciences, Clarendon Press, Oxford 1950. 